\title{
Tratamiento de anemia aplásica severa adquirida en pacientes pediátricos con inmunosupresión y trasplante alogénico de precursores hematopoiéticos
}

\author{
Francisco Barriga $C^{1}$, Angélica W ietstruck $\mathrm{P}^{1}$, Anna Becker $\mathrm{K}^{3}$, \\ Pamela Zúñiga $C^{1}$, Pelayo Besa De $C^{2}$, Manuel Alvarez $Z^{2}$, \\ Mauricio 0 cqueteau $T^{2}$, Pablo Bertín $C^{2}$. \\ Treatment of acquired severe aplastic \\ anemia in pediatric patients with \\ immunosupression and allogeneic \\ stem cell hematopoyetic transplant
}

Background: Severe acquired aplastic anemia (SAA) is an uncommon disease of childhood. Patients with SAA receive supportive care with transfusions and timely treatment of opportunistic infections, along with specific therapies, which may be allogenic stem cell transplantation(SCT) from a matched sibling or immunosupressive therapy (IT). Aim: To report the experience in the management of SAA. Patients and methods: Twenty five children with acquired SAA were treated from July 1992 to September 2005. Patients with full matched sibling donors received allogenic SCT after conditioning with a cyclophosphamide containing regimen. The other patients received immune suppression with cyclosporine plus methylprednisolone $(n=18)$ plus ATG $(n=17)$. All received supportive care until recovery of hematopoietic function. Those who had severe opportunistic infections at diagnosis or did not respond to two cycles of ATG were evaluated for unrelated donor SCT. Results: Seven patients received sibling donor SCT and $18 \mathrm{IT}$, which was repeated in six. Three patients received mismatched related (1) or unrelated (2) SCT. Nineteen patients survived with a median follow up time of 4 years, 14 with full hematologic recovery. Six patients died: four due to infections after IT or SCT, one due to intracranial hemorrhage and one with secondary myelodysplasia 12 years after IT. Conclusions: Most children with SAA can be treated successfully with sibling donor SCT or IT. Patients without a histocompatible sibling who fail to respond to IS have a worse prognosis (Rev Méd Chile 2007; 135: 1421-28).

(Key words: Anemia, aplastic; Immunosupression; Stem cell transplantation)

Recibido el 30 de agosto 2006. Aceptado el 29 de mayo 2007.

Departamentos de Pediatría ${ }^{1}$ y Hematología-Oncología ${ }^{2}$, Pontificia Universidad Católica de Chile. Servicio de Pediatría Hospital Sótero del Rio ${ }^{3}$, Santiago de Chile.

Correspondencia a: Dr. Francisco Barriga. Departamento de Pediatría, Escuela de Medicina, Pontificia Universidad Católica de Chile. Lira 85, 5ำ piso. Santiago, Chile. Fax: 638-4307. E mail: fbarriga@med.puc.cl 
$\mathrm{L}$ a anemia aplásica severa (AAS) adquirida es una enfermedad poco frecuente en el grupo pediátrico, caracterizada por insuficiencia de la función hematopoiética que resulta en anemia hiporregenerativa $(\mathrm{Hgb}<8 \mathrm{gr} / \mathrm{dl}$, reticulocitos $<1 \%)$, neutropenia $\left(<500 / \mathrm{mm}^{3}\right)$ y trombocitopenia $\left(<20.000 / \mathrm{mm}^{3}\right)^{1,2}$. La incidencia estimada es de 1 a 6 casos por millón de habitantes ${ }^{3}$. Se desconoce la etiología de la AAS adquirida, si bien se ha asociado ocasionalmente a la exposición a fármacos ${ }^{4}$, o a hepatitis $\mathrm{B}^{5}$. Recientemente se han descrito alteraciones genéticas que predisponen a la aparición de esta enfermedad ${ }^{6,7}$ y también se postula que pacientes con formas congénitas de aplasia medular pueden debutar sin otras alteraciones típicas ${ }^{8,9}$. Se especula sobre posibles mecanismos autoinmunes en su patogenia, debido a la respuesta clínica que se observa en pacientes tratados con terapia inmunosupresora ${ }^{10,11}$.

El manejo actual de la AAS en el grupo pediátrico tiene dos aspectos: terapia de soporte y terapia específica ${ }^{12}$. La terapia de soporte se realiza mediante transfusiones de productos sanguíneos, prevención y tratamiento de infecciones y uso de factores estimulantes de colonias hematopoiéticas. La terapia específica comprende: a) Trasplante de precursores hematopoiéticos (TPH) alogénico de un donante hermano compatible, considerado el tratamiento de elección de la AAS en el paciente pediátrico y adulto joven ${ }^{12,13}$. Más de $80 \%$ de los pacientes trasplantados tienen recuperación completa de función medular. b) Terapia inmunosupresora combinada con corticoides, globulina antitimocítica y un inhibidor de la calcineurina (ciclosporina) por un período de tres o más meses junto con la terapia de apoyo ${ }^{14-16,26}$. Aproximadamente 60\%-80\% de los pacientes tratados así tienen mejoría de su función medular, la que puede ser completa o parcial. c) TPH de donante alternativo: se reserva para los pacientes que sin tener un donante emparentado compatible se mantienen dependientes de transfusiones o con infecciones oportunistas repetidas después de 6 meses de terapia inmunosupresora combinada ${ }^{17,18}$. La mayoría de estos trasplantes se han hecho con donantes adultos voluntarios no emparentados obtenidos a través de registros de donantes y ocasionalmente con donantes familiares no idénticos ${ }^{19,20}$.

El objetivo del presente trabajo es presentar la experiencia en el manejo de la AAS adquirida en pacientes pediátricos tratados en dos instituciones entre 1992 y 2006 utilizando cuidado de apoyo y terapia específica según un esquema común para analizar los resultados de las diversas modalidades y buscar factores de riesgo tanto biológicos como relacionados a la terapia que permitan evaluar precozmente el pronóstico de este grupo de pacientes y analizar, a su vez, las causas de mortalidad en ellos.

\section{PACIENTES Y MÉTODO}

Diagnóstico. 25 pacientes pediátricos (rango edad 2 a 18 años) con el diagnóstico de AAS adquirida fueron tratados en nuestras instituciones desde julio 1992 a enero 2006. El diagnóstico se confirmó mediante mielograma y biopsia de médula, con estudio de citogenética normal en todos los casos. A los pacientes con antecedentes familiares de aplasia medular se les realizó el examen de sensibilidad cromosómica al diepoxibutano (DEB) para descartar anemia de Fanconi ${ }^{21}$.

Terapia de apoyo. Los pacientes recibieron terapia transfusional para mantener un recuento de plaquetas mayor o igual a $20.000 / \mathrm{mm}^{3}$ y una hemoglobina mayor o igual a $8,0 \mathrm{~g} / \mathrm{dl}$. A los pacientes con fiebre $\left(>38,0^{\circ} \mathrm{C}\right)$ se les tomó hemocultivo y fueron tratados con antibióticos de amplio espectro, además de recibir profilaxis inicial con aciclovir y fluconazol. Se pesquisaron y trataron precozmente con antibióticos de amplio espectro todas las infecciones oportunistas con demostración clínica o de laboratorio. Se realizaron estudios de HLA A,B y DR a los pacientes y sus hermanos.

Terapia específica. Los pacientes se manejaron según el esquema de la Figura 1, el que fue adoptado desde el principio coincidiendo con el inicio de nuestro programa de TPH alogénico. Brevemente, los pacientes con hermano donante compatible recibieron trasplante y los que no lo tenían tratamiento inmunosupresor. A los padres de todos los pacientes se les explicó con claridad la naturaleza de la enfermedad, el esquema terapéutico a seguir en caso de identificar o no un donante hermano(a) compatible y los riesgos asociados a cualquiera de las modalidades terapéuticas elegidas como las probabilidades de 


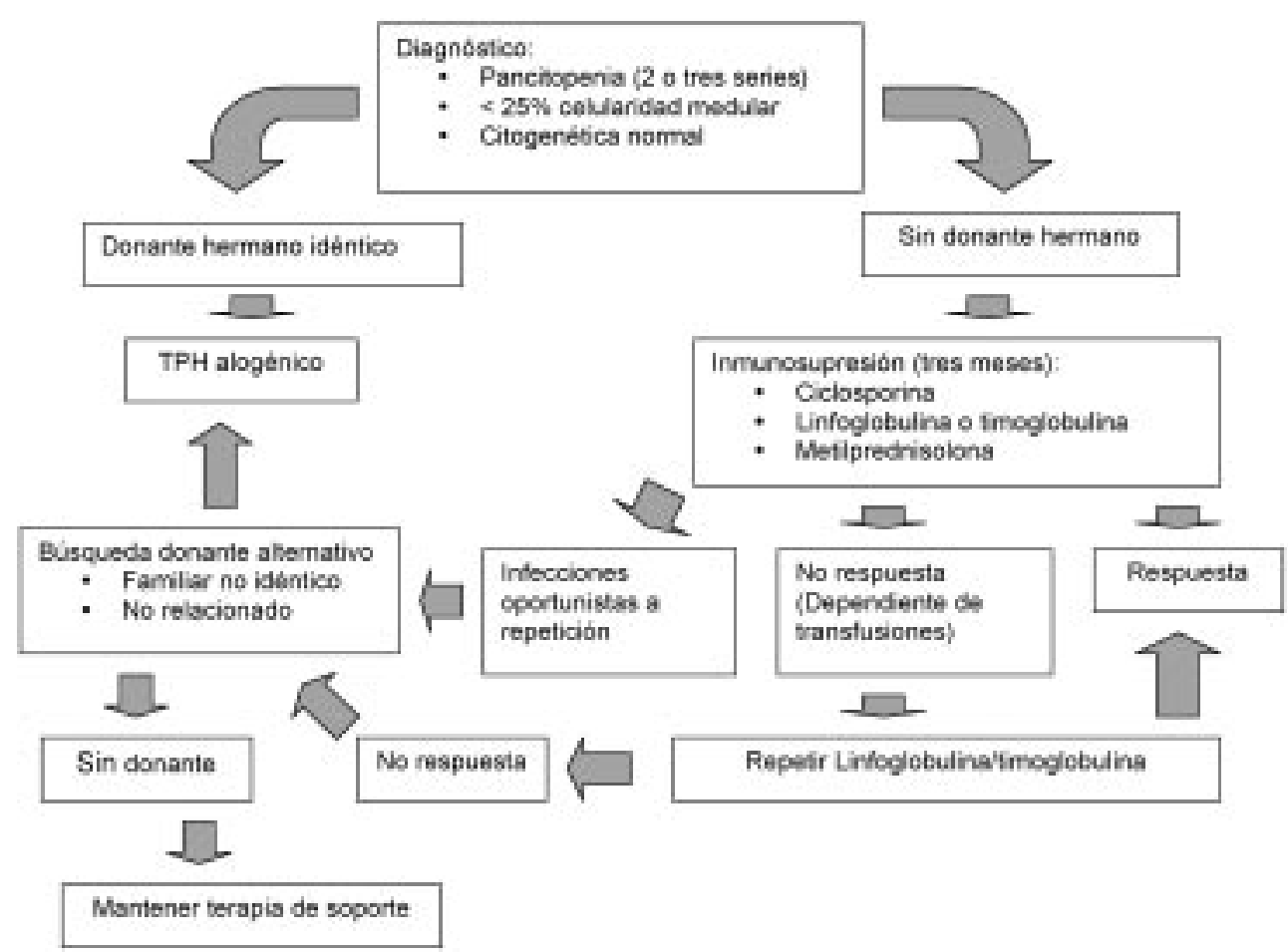

Figura 1. Esquema de tratamiento de anemia aplásica severa

éxito de la misma. No hubo exclusión de pacientes con AAS del análisis durante el tiempo del estudio ni asignación de la modalidad terapéutica elegida por criterios económicos o extra médicos.

Los pacientes trasplantados recibieron condicionamiento con ciclofosfamida +/-busulfán +/radioterapia nodal o corporal total $(\mathrm{RNT})^{22}$. La inmunosupresión consistió en globulina antilinfocítica (ATG: Linfoglobulina o Timoglobulina, Pasteur Merieux), metilprednisolona $2 \mathrm{mg} / \mathrm{kg}$ día por x 5 días para pasar a prednisona a igual dosis, $\mathrm{y}$ ciclosporina 3 a $5 \mathrm{mg} / \mathrm{kg} /$ día para mantener niveles basales alrededor de $200 \mathrm{ng} / \mathrm{ml}^{23}$. Los pacientes trasplantados recibieron profilaxis para enfermedad de injerto versus huésped (EICH) con ciclosporina y metotrexato ${ }^{24}$. Todos continuaron con terapia de apoyo transfusional y tratamiento precoz de infecciones oportunistas hasta la recuperación medular. Los pacientes que recibieron IS y persistieron dependientes de transfusiones a tres meses de la dosis de ATG, recibieron un nuevo curso de ATG con el mismo preparado o el alternativo. Aquellos que persistieron sin respuesta después del segundo curso o aquellos que presentaron infecciones oportunistas a repetición al diagnóstico fueron evaluados para TPH de donante alternativo (familiar no idéntico o donante no relacionado).

Estadística. La sobrevida del grupo completo de pacientes se calculó por método de Kaplan y Meier. Comparamos la sobrevida entre pacientes que tenían donante hermano o tuvieron una respuesta completa a IS (riesgo favorable) y los demás (riesgo desfavorable), mediante test de chi cuadrado.

\section{Resultados}

Características de los pacientes. Todos los pacientes cumplieron los criterios diagnósticos de AAS. 
Un paciente debutó tres semanas después del uso de cloranfenicol por una infección gastrointestinal. Dos pacientes, que fueron diagnosticados a los 4 y 7 años, eran hermanos y tenían el antecedente de una hermana mayor fallecida de AAS. La determinación de sensibilidad cromosómica al DEB descartó anemia de Fanconi en ambos. Cuatro pacientes se presentaron con neutropenia menor de $200 / \mathrm{mm}^{3}$, fiebre e infecciones oportunistas. Veinte pacientes requirieron terapia transfusional con plaquetas y glóbulos rojos desde su inicio.

Esquemas de tratamiento. Siete pacientes tuvieron un hermano(a) HLA idéntico e iniciaron condicionamiento para TPH entre 7 días y un año desde el diagnóstico (Tabla 1). Una paciente demostró recuperación espontánea de neutrófilos en el tercer día de condicionamiento con ciclofosfamida, por lo que se difirió el TPH. Seis pacientes, incluyendo la que no alcanzó a recibir TPH, tuvieron recuperación completa y permanente de su función hematopoiética. Un paciente tuvo EICH severo cutáneo e intestinal y se mantuvo hospitalizado por 8 meses para manejo de complicaciones asociadas (septicemia, dolor abdominal, oclusión intestinal, aspergilosis) hasta la resolución del EICH. Una paciente que recibió su TPH 8 meses después del diagnóstico, habiendo recibido numerosas transfusiones, tuvo prendimiento de la serie granulocítica con demostración por quimerismo de cromosomas sexuales, pero no reconstituyó serie roja ni plaquetaria $^{25}$. Diecisiete pacientes recibieron IS completa y uno recibió ciclosporina y metilprednisolona (Tabla 2). Seis pacientes obtuvieron recuperación completa entre 2 y 8 meses del diagnóstico después del primer curso de IS. Seis recibieron un segundo curso por falta de respuesta y de ellos 3 recuperaron hematopoiesis. Tres pacientes recibieron TPH de donante alternativo: uno de sangre de cordón de donante no emparentado por infecciones oportunistas severas al diagnóstico y dos por no-respuesta a 2 cursos de ATG. Uno de ellos recibió TPH de donante adulto voluntario en Seattle (USA) y otro recibió TPH de sangre de cordón seguido de TPH de su abuela materna, compatible en 8 de 10 antígenos HLA estudiados por alta resolución.

Causas de mortalidad y sobrevida. Dos pacientes fallecieron, por shock séptico y hemorragia, respectivamente, 25 días y 6 meses después de recibir IS. Dos pacientes fallecieron por complicaciones posteriores a TPH de donante alternativo (infección por hongos sin prendimiento a los 40 días, EICH

\section{Tabla 1. C aracterísticas y resultados de 7 pacientes con donante hermano histocompatible que recibieron trasplante de médula ósea.}

\begin{tabular}{|lcccccc|}
\hline $\begin{array}{l}\text { Sexo/ } \\
\text { Edad }\end{array}$ & $\begin{array}{c}\text { Dependiente } \\
\text { de transfusión } \\
\text { al diagnóstico }\end{array}$ & $\begin{array}{c}\text { Infección } \\
\text { oportunista } \\
\text { al diagnóstico }\end{array}$ & $\begin{array}{c}\text { Tiempo } \\
\text { Diagnóstico } \\
\text { a TPH }\end{array}$ & Respuesta & EICH & Resultado \\
\hline $\mathrm{F} / 3$ & SI & NO & 8 meses & RHP & NO & Fallece (hemorragia cerebral) \\
$M / 6$ & SI & NO & 2 meses & RHC & NO & En remisión 2,1 años \\
$M / 11$ & NO & NO & 4 años & RHC & NO & En remisión 4,6 años \\
$M / 16$ & SI & SI & 15 días & RHC & $*$ & En remisión 4,7 años \\
$M / 9$ & SI & NO & 2 meses & RHC & Grado IV & En remisión sin EICH 5 años \\
$M / 13$ & SI & NO & 3 meses & RHC & NO & En remisión 7,2 años \\
F $/ 4$ & SI & NO & 4 meses & RHC & NO & En remisión 8 años \\
\hline
\end{tabular}

Dependiente de transfusión: sangramiento mucoso activo o Hgb $<8$ sin transfusión.

Infección oportunista: mucositis herpética

*No alcanzó a recibir TPH por recuperación espontánea durante condicionamiento.

RHC: Reconstitución hematopoiética completa

RHP: Reconstitución hematopoiética parcial (anemia y trombocitopenia severas) 


\section{Tabla 2. Características y resultado de 18 pacientes sin donante hermano que recibieron tratamiento IS}

\begin{tabular}{|c|c|c|c|c|c|c|}
\hline $\begin{array}{l}\text { Sexo/ } \\
\text { Edad }\end{array}$ & $\begin{array}{l}\text { Dependiente } \\
\text { de transfusión } \\
\text { al diagnóstico }\end{array}$ & $\begin{array}{c}\text { Infección } \\
\text { oportunista } \\
\text { al diagnóstico }\end{array}$ & $\begin{array}{c}\text { Ciclos de } \\
\text { ATG } \\
\text { Alternativo? }\end{array}$ & Respuesta & $\begin{array}{c}\text { TPH } \\
\text { Donante }\end{array}$ & Resultado \\
\hline $\mathrm{F} / 11$ & SI & SI & 1 & $\mathrm{NO}$ & & Fallece (shock séptico) \\
\hline $\mathrm{M} / 2$ & SI & SI & 1 & $\mathrm{NO}$ & SI & Fallece (aspergilosis) sin prendimiento \\
\hline $\mathrm{F} / 12$ & SI & $\mathrm{NO}$ & 1 & RHC & & En remisión 1 año \\
\hline $\mathrm{M} / 5$ & SI & SI & 1 & $\mathrm{NO}$ & & Fallece (hemorragia cerebral) \\
\hline $\mathrm{M} / 8$ & NO & NO & 1 & RHP & & Vivo independiente de transfusiones 3,6 años \\
\hline $\mathrm{M} / 9$ & SI & NO & 1 & $\mathrm{RHC}$ & & En remisión 5,8 años \\
\hline $\mathrm{F} / 12$ & SI & NO & 1 & $\mathrm{RHC}$ & & En remisión 8,8 años \\
\hline $\mathrm{M} / 11$ & SI & $\mathrm{NO}$ & 1 & $\mathrm{RHC}$ & & En remisión 9,5 años \\
\hline $\mathrm{M} / 7$ & NO & $\mathrm{NO}$ & 1 & RHP & & Fallece (mielodisplasia 12,6 años) \\
\hline $\mathrm{M} / 12$ & NO & $\mathrm{NO}$ & 1 & $\mathrm{RHC}$ & & En remisión 5,5 años \\
\hline $\mathrm{F} / 11$ & NO & $\mathrm{NO}$ & 1 & RHC & & En remisión 4,3 años \\
\hline $\mathrm{M} / 11$ & SI & NO & 1 & RHP & & Vivo independiente de transfusiones, 2,1 años \\
\hline $\mathrm{M} / 6$ & SI & $\mathrm{NO}$ & 2 & $\mathrm{NO}$ & & Vivo dependiente de transfusiones 2 años \\
\hline $\mathrm{M} / 11$ & SI & $\mathrm{NO}$ & 2 & $\mathrm{NO}$ & SI & Fallece (EICH) \\
\hline $\mathrm{M} / 14$ & SI & $\mathrm{NO}$ & 2 & $\mathrm{RHC}$ & & En remisión 4,7 años \\
\hline $\mathrm{M} / 8$ & SI & $\mathrm{NO}$ & 2 & $\mathrm{NO}$ & SI & Vivo con EICH 7,6 años \\
\hline $\mathrm{F} / 12$ & SI & $\mathrm{NO}$ & 2 & $\mathrm{RHC}$ & & En remisión 9,4 años \\
\hline $\mathrm{M} / 2$ & SI & $\mathrm{NO}$ & 2 & $\mathrm{NO}$ & & Vivo con enfermedad 2,1 años \\
\hline
\end{tabular}

Dependiente de transfusión: sangramiento mucoso activo o $\mathrm{Hgb}<8$ sin transfusión.

Infección oportunista: bacteremia, mucositis herpética, sinusitis por aspergilosis

RHC: Reconstitución hematopoiética completa

RHP: Reconstitución hematopoiética parcial (trombocitopenia leve a moderada, anemia leve)

agudo a los 3 meses) y una paciente por hemorragia después de prendimiento parcial (sólo neutrófilos), 4 meses post TPH de hermano ${ }^{25}$. Un paciente tuvo respuesta parcial a ATG y desarrolló mielodisplasia de alto riesgo (anemia refractaria con exceso de blastos) 12 años después de su tratamiento, falleciendo por toxicidad pulmonar e infecciones en la inducción de la remisión. En total 6 pacientes fallecieron, tres de los cuales recibieron TPH. Cuatro de ellos por complicaciones de la enfermedad (shock séptico y hemorragia) y dos por complicaciones de TPH de donante no emparentado. Con una mediana de seguimiento de 4 años, la sobrevida para el grupo es de 79,4\% (Figura 2). Catorce pacientes tienen recuperación hematopoiética completa y cinco se mantienen con insuficiencia medular, dos sin respuesta a IS, dos por recidiva tras IS y otro por EICH crónico después de TPH de donante familiar no idéntico.
Análisis de factores pronósticos. Identificamos los siguientes factores de pronóstico favorable: TPH de donante hermano idéntico y respuesta completa a IS ${ }^{23}$. Los pacientes con estas características ( $n$ =17) tuvieron una sobrevida por método Kaplan Meier a 5 años del 93,7\%, mientras que los demás $(\mathrm{n}=8)$ una sobrevida de $50 \%(\mathrm{p}=0,009$, Figura 3$)$. Tres de los 4 pacientes con neutropenia inicial de $<200$ neutrófilos $/ \mathrm{mm}^{3}$ fallecieron por enfermedad, pero solo uno tuvo donante hermano y se corrigió espontáneamente durante el condicionamiento del trasplante.

\section{DisCUSIÓN}

Presentamos los resultados de la serie más numerosa comunicada de pacientes pediátricos con AAS adquirida en Chile y la primera que incorpora el TPH de rutina en el manejo inicial de estos 


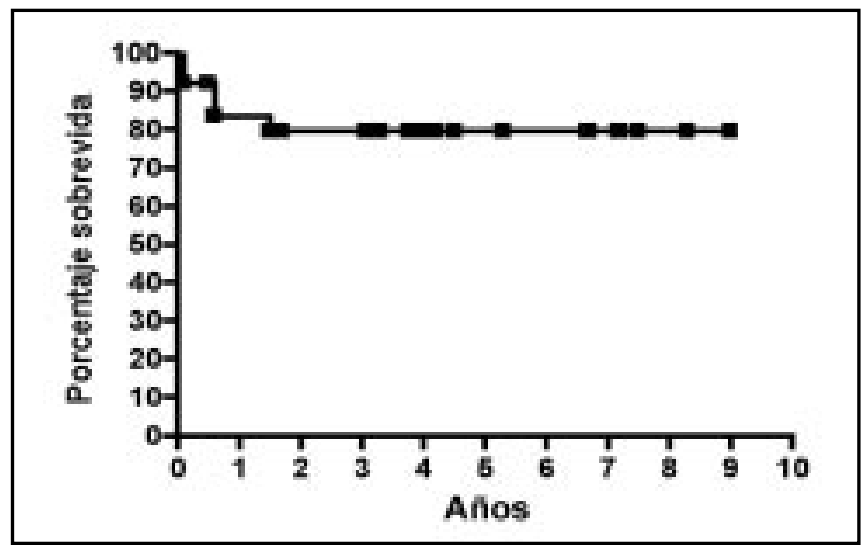

Figura 2. Sobrevida por método Kaplan-Meier de 25 pacientes pediátricos con anemia aplásica severa

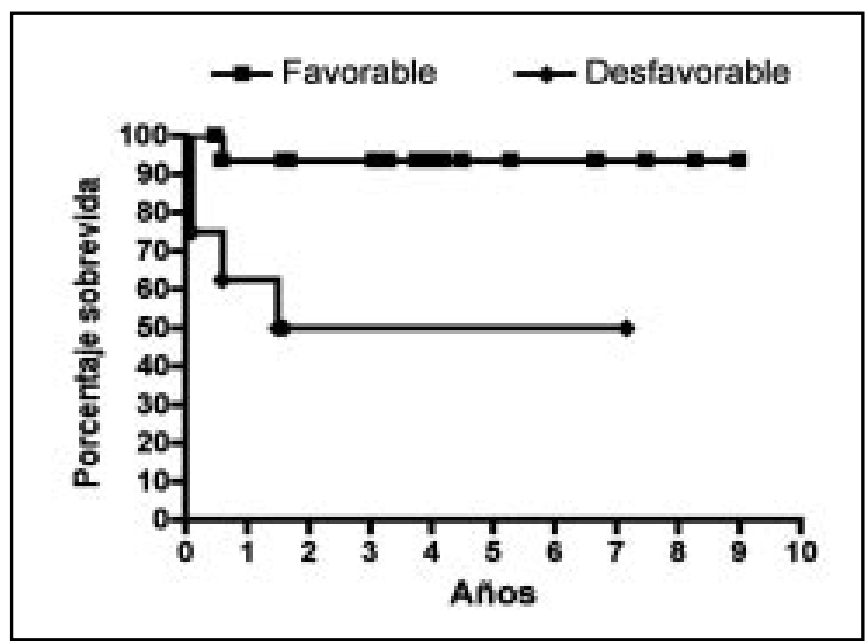

Figura 3. Sobrevida por método Kaplan-Meier de pacientes con características favorables (trasplante hermano compatible o respuesta completa a inmunosupresión) y desfavorables (los demás)

pacientes. Una comunicación anterior de Tordecilla et al, describió la experiencia en 7 casos, dos de los cuales tuvieron AAS congénita y uno de los cuales fue trasplantado ${ }^{26}$. Nuestra serie enfatiza el curso impredecible y a veces complejo de esta enfermedad de mecanismos patogénicos no precisados, y da una idea de los resultados esperables con los tratamientos actuales.

El TPH de donante hermano idéntico es considerado por la mayoría como el tratamiento de elección de esta patología, si bien la mayoría de los pacientes carecen de dicho donante. No obstante, la mejoría en la respuesta obtenida en la actualidad con la terapia IS ha llevado a muchos a cuestionar esta prioridad, debido al riesgo inherente de mortalidad que tiene dicho procedimiento por complicaciones del mismo, fundamentalmente la $\mathrm{EICH}^{27}$. Sin embargo, varios aspectos afirman la elección del TPH como primera línea: lo impredecible de la respuesta completa a la IS, que es cercana a $60 \%$ en la mayoría de las series reportadas, y la posibilidad de evolución clonal de la enfermedad 
hacia una mielodisplasia o leucemia aguda, la que es significativamente mas frecuente en pacientes tratados con IS ${ }^{13,23,28,29}$. En nuestra serie, 6 de los 7 pacientes (85\%) que recibieron TPH de hermano(a) tuvieron recuperación completa, versus 9 de los 18 (50\%) que recibieron IS ( $\mathrm{p}=\mathrm{NS}$ ).

El desafío actual en el manejo de estos pacientes lo constituyen aquellos que sin tener donante hermano(a) no responden a IS, quedando dependientes de terapia transfusional a permanencia. Estos pacientes pueden sobrevivir por largos períodos de tiempo cuando se dispone de un banco de sangre que los pueda transfundir a demanda, pero su calidad de vida es muy pobre y permanecen en riesgo de tener infecciones oportunistas, hemorragias severas, a pesar de las trasfusiones, o que aparezca evolución clonal hacia mielodisplasia o leucemia aguda. En estos pacientes el TPH de donantes alternativos (familiares no idénticos o donantes no emparentados) ha conseguido remi-

\section{REFERENCIAS}

1. Kurre P, Johnson FL, Deeg HJ. Diagnosis and treatment of children with aplastic anemia. Pediatr Blood Cancer 2005; 45: 770-80.

2. Bagby GC, Lipton JM, Sloand EM, Schiffer CA. Marrow failure. Hematology (Am Soc Hematol Educ Program) 2004; 318-36.

3. Locasciulli A. Acquired aplastic anemia in children: incidence, prognosis and treatment options. Paediatr Drugs 2002; 4: 761-6.

4. Maluf EM, Pasquini R, Eluf Jn, Kelly J, Kaufman DW. Aplastic anemia in Brazil: incidence and risk factors. Am J Hematol 2002; 71: 268-74.

5. Brown Ke, Tisdale J, Barrett AJ, Dunbar CE, Young NS. Hepatitis-associated aplastic anemia. N Engl J Med 1997; 336: 1059-64.

6. Kapustin SI, Popova TI, Lyshchov AA, Imyanttov EN, Blinov MN, ABdulKadYrov KM. HLA-DR4-Ala74 beta is associated with risk and poor outcome of severe aplastic anemia. Ann Hematol 2001; 80: 66-71.

7. Sutton Jf, Stacey M, Kearns WG, Rieg tS, Young NS, LIu JM. Increased risk for aplastic anemia and myelodysplastic syndrome in individuals lacking glutathione S-transferase genes. Pediatr Blood Cancer 2004; 42: 122-6. sión definitiva de la enfermedad pero con resultados mas bien modestos y una alta mortalidad asociada al procedimiento ${ }^{20}$. Esta a su vez puede estar relacionada al tiempo prolongado de evolución desde el diagnóstico al trasplante, por acúmulo de transfusiones múltiples e infecciones oportunistas repetidas. No hay consenso acerca del mejor momento para considerar TPH de donante alternativo a pacientes con AAS, ni cual sería el donante óptimo para estos pacientes.

En resumen, la AAS adquirida es una enfermedad infrecuente en pacientes pediátricos, de curso impredecible y manejo complejo. Los pacientes con hermano(a) compatible idéntico se benefician con TPH, los que no tienen un donante deben recibir IS al mismo tiempo que se mantiene una terapia de apoyo intensiva. Estos pacientes deben permanecer en seguimiento por largos períodos de tiempo para descartar la evolución clonal de la enfermedad.

8. Fogarty Pf, Yamaguchi H, Wiestner A, Baerlocher GM, SloAnd E, Zeng WS, ET AL. Late presentation of dyskeratosis congenita as apparently acquired aplastic anaemia due to mutations in telomerase RNA. Lancet 2003; 362(9396): 1628-30.

9. Giampietro PF, Verlander PC, Davis JG, Auerbach AD. Diagnosis of Fanconi anemia in patients without congenital malformations: an international Fanconi Anemia Registry Study. Am J Med Genet 1997; 68: 58-61.

10. Bacigalupo A, Valle M, Podesta M, Pitto A, Zocchi E, De Flora A, Et al. T-cell suppression mediated by mesenchymal stem cells is deficient in patients with severe aplastic anemia. Exp Hematol 2005; 33: 819-27.

11. Nakao S, Feng X, Sugimori C. Immune pathophysiology of aplastic anemia. Int J Hematol 2005; 82(3): 196-200.

12. Sanders Je, Storb R, Anasetti C, Deeg HJ, Doney K, Suldivan KM, ET AL. Marrow transplant experience for children with severe aplastic anemia. Am J Pediatr Hematol Oncol 1994; 16: 43-9.

13. Doney K, Leisenring W, Storb R, Appelbaum FR. Primary treatment of acquired aplastic anemia: outcomes with bone marrow transplantation and immunosuppressive therapy. Seattle Bone Ma- 
rrow Transplant Team. Ann Intern Med 1997; 126: $107-15$.

14. Frickhofen N, Heimpel H, Kaltwasser JP, SchrezenMEIER H. Antithymocyte globulin with or without cyclosporin A: 11-year follow-up of a randomized trial comparing treatments of aplastic anemia. Blood 2003; 101: 1236-42.

15. Bacigalupo A, Bruno B, Saracco P, Di Bona E, Locasciulli A, Locatelli F, et al. Antilymphocyte globulin, cyclosporine, prednisolone, and granulocyte colony-stimulating factor for severe aplastic anemia: an update of the GITMO/EBMT study on 100 patients. European Group for Blood and Marrow Transplantation (EBMT) Working Party on Severe Aplastic Anemia and the Gruppo Italiano Trapianti di Midolio Osseo (GITMO). Blood 2000; 95: 1931-4.

16. Bekassy AN, locasciulli A, Marsh JC, Socie G, Fuehrer M, Passweg J. Immunosuppression with ALG and CsA is first line treatment in children with SAA lacking an HLA identical sibling. Pediatr Blood Cancer 2005; 45: 359-60; author reply 361-2.

17. Vassiliou GS, Webb DK, Pamphilon D, Knapper S, VEYS PA. Improved outcome of alternative donor bone marrow transplantation in children with severe aplastic anaemia using a conditioning regimen containing low-dose total body irradiation, cyclophosphamide and Campath. Br J Haematol 2001; 114: 701-5.

18. Gupta V, Ball SE, Sage D, Ortin M, Freires M, Gordon-Smith EC, et al. Marrow transplants from matched unrelated donors for aplastic anaemia using alemtuzumab, fludarabine and cyclophosphamide based conditioning. Bone Marrow Transplant 2005; 35: 467-71.

19. Locatelli F, Porta F, Zecca M, Pedrazzoli P, MaccaRio R, Giani S, ET al. Successful bone marrow transplantation in children with severe aplastic anemia using HLA-partially matched family donors. Am J Hematol 1993; 42: 328-33.

20. Deeg HJ, Seidel K, Casper J, Anasetti C, Davies S, GAJEWESKI JL, ET AL. Marrow transplantation from unrelated donors for patients with severe aplastic anemia who have failed immunosuppressive therapy. Biol Blood Marrow Transplant 1999; 5: 243-52.

21. Auerbach AD. Fanconi anemia diagnosis and the diepoxybutane (DEB) test. Exp Hematol 1993; 21: 731-3.
22. Gaziev D, Giardini C, Galimberti M, Lucarelli G, Angelucci E, Polchi P, et al. Bone marrow transplantation for transfused patients with severe aplastic anemia using cyclophosphamide and total lymphoid irradiation as conditioning therapy: long-term follow-up from a single center. Bone Marrow Transplant 1999; 24: 253-7.

23. Rosenfeld S, Follmann D, Nunez O, Young NS. Antithymocyte globulin and cyclosporine for severe aplastic anemia: association between hematologic response and long-term outcome. JAMA 2003; 289: 1130-5.

24. Locatelli F, Bruno B, Zecca M, Van-Lint MT, McCann S, Arcese W, et al. Cyclosporin A and short-term methotrexate versus cyclosporin A as graft versus host disease prophylaxis in patients with severe aplastic anemia given allogeneic bone marrow transplantation from an HLA-identical sibling: results of a GITMO/EBMT randomized trial. Blood 2000; 96: 1690-7.

25. Barriga FJ, Legues ME, Bertín P. Selective engraftment of the granulocyte compartment after allogeneic bone marrow transplantation in a patient with severe aplastic anemia. J Pediatr Hematol Oncol 1996; 18: 216-7.

26. Tordecilla CJ, Campbell M, Joannon P. Anemia Aplástica: Experiencia con 7 casos. Rev Chil Pediatr 2003; 74: 179-186.

27. Lawlor ER, Anderson RA, Davis JH, Fryer CJ, Pritchard SL, Rogers PC, ET AL. Immunosuppressive therapy: a potential alternative to bone marrow transplantation as initial therapy for acquired severe aplastic anemia in childhood? J Pediatr Hematol Oncol 1997; 19: 115-23.

28. Gillio AP, Boulad F, Small TN, Kernan NA, Reyes B, Childs BH, ET AL. Comparison of long-term outcome of children with severe aplastic anemia treated with immunosuppression versus bone marrow transplantation. Biol Blood Marrow Transplant 1997; 3: 18-24.

29. Fuhrer M, Burdach S, Ebell W, Gadner H, Haas R, Harbott J, et al. Relapse and clonal disease in children with aplastic anemia (AA) after immunosuppressive therapy (IST): the SAA 94 experience. German/Austrian Pediatric Aplastic Anemia Working Group. Klin Padiatr 1998; 210: 173-9. 